\title{
Aggressive Resection of Perihilar Cholangiocarcinoma: A Case Report and Literature Review
}

\author{
Jijun Shan, Changxian Li and Xiangcheng $\mathrm{Li}^{*}$ \\ Hepatobiliary Center, The First Affiliated Hospital of Nanjing Medical University, Nanjing, China
}

*Corresponding author: Xiangcheng Li, Hepatobiliary Center, The First Affiliated Hospital of Nanjing Medical University, Nanjing, China

\section{ARTICLE INFO}

Received: 画 May 10, 2021

Published: 豐 May 21, 2021

Citation: Jijun Shan, Changxian Li, Xiangcheng Li. Aggressive Resection of Perihilar Cholangiocarcinoma: A Case Report and Literature Review. Biomed J Sci \& Tech Res 36(1)-2021. BJSTR. MS.ID.005785.

\begin{abstract}
Perihilar cholangiocarcinoma is one of the most challenging diseases and with no obvious symptoms at early stage, so it is difficult for surgeons to reach negative margin during surgery. Although the diagnostic accuracy of both computed tomography and magnetic resonance imaging have improved, histopathology can give the definitive diagnosis. And the recommended treatment is resection-based-multidisciplinary treatment. Now we present a case of perihilar cholangiocarcinoma.
\end{abstract}

Abbreviations: iCCA: Intrahepatic; pCCA: Perihilar; dCCA: Distal; PSC: Primary Sclerosing Cholangitis; BC: Bismuth Corlette; CT: Computed Tomography; PVE: Portal Vein Embolization; PVL: Portal Vein Ligation

\section{Introduction}

Cholangiocarcinoma is a rare but aggressive tumor, accounting for $10-15 \%$ of all hepatobiliary cancers and $3 \%$ of all gastrointestinal tumors [1], arising from varying locations within the biliary tree. It can be divided into intrahepatic(iCCA), perihilar(pCCA), and distal(dCCA) cholangiocarcinoma according to anatomical location. Perihilar disease represents about 50\%, distal disease $40 \%$, and intrahepatic disease less than $10 \%$ of cholangiocarcinoma cases [2]. Known risk factors include primary sclerosing cholangitis (PSC), liver fluke infestation (Clonorchis sinensis and Opisthorchis viverrini) and hepatolithiasis, but most cases are sporadic without an apparent inciting factor [3]. Perihilar cholangiocarcinoma presents with obstructive jaundice, unless only the left or right duct is intimately involved. Tea colored urine, clay colored stools, anorexia, weight loss, vague abdominal pain, and pruritis are common complaints. So far, surgical resection remains the only potentially curative treatment for long-term survival and liver transplantation after neoadjuvant chemoradiation is restricted to a subset of patients with early stage pCCA [4]. However, for patients with advanced-stage or unresectable disease, multidisciplinary treatment approach is key to achieve optimal patient outcomes.

\section{Case Presentation}

The 61-year-old female with cholecystectomy and right mastectomy history presented with dull pain in the upper right quadrant and weight loss. CT reveals: Beside hepatic caudate lobe, there is a small patch of uneven enhancement of soft tissue density at the confluence of the left and right hepatic ducts with obvious dilatation of intrahepatic bile ducts. The upper part of the common bile duct is dilated, and the walls of the upper part of the common bile duct and the remaining cystic duct are slightly thickened and strengthened. Hilar lymph nodes slightly enlarged. According to the condition, we perform a surgery for her. Intraoperative exploration: The upper part of the common bile duct is dilated. A lump in the caudate lobe of the liver can be palpable, about $3 * 2 \mathrm{~cm}$ in size, with a hard texture, involving the bifurcation of the left and right hepatic ducts, and involving the right hepatic duct. Several enlarged lymph nodes were touched at the hilar, next to the common hepatic artery, and behind the head of the pancreas. The tumor involves the junction of the left and right branches of the portal vein and the distal end of the right branch, and part of the vena cava is significantly affected by the tumor. Intraoperative diagnosis: hilar cholangiocarcinoma 
(type IIIa), radical operation of hilar cholangiocarcinoma was performed (Right hemiliver combined with full caudate lobectomy + hilar lymph node dissection + portal vein reconstruction + cavity Vein reconstruction + left hepatic duct jejunostomy). Pathological diagnosis is cholangiocarcinoma and margin is negative. After the operation, the patient was discharged safely and went to the oncology department for further treatment.

\section{Discussion}

Perihilar cholangiocarcinoma is a disease of advanced age with an unclear etiology, most frequently found in Southeast Asia and relatively rare in Western countries. It is the most common primary tumor of the biliary tract although it accounts for only $2 \%$ of all human malignancies.

\section{Classification}

For perihilar cholangiocarcinoma, Bismuth and Corlette described their criteria for categorizing perihilar bile duct cancers in 1975 [5]. The local extent of the tumor is most commonly described using the Bismuth-Corlette (BC) system. This classification provides information regarding the level and longitudinal extent of the tumor relative to the biliary confluences. Tumors are classified into type I (tumors involving only the common hepatic duct below the first confluence), type II (tumors involving the first confluence without involvement of the second confluences), type III (tumors involving either right [IIIA] or left [IIIB] second confluences), or type IV (tumors involving both right and left second confluences) [6].

\section{Diagnosis}

Cholangiocarcinoma is a primary malignant tumor originating from the bile duct epithelium that is mostly adenocarcinoma at histologic examination [7]. Many advanced patients always present with obstructive jaundice, tea colored urine, clay colored stools, anorexia, weight loss, vague abdominal pain, pruritis and upper abdomen dull pain. With the improvement of medical imaging accuracy, imaging plays a critical role in diagnosis and making treatment planning. Imaging tools including mainly multidetector computed tomography (CT), MRI with MRCP, and PET have been demonstrated to be useful in establishing the correct diagnosis and in determining respectability with a high negative predictive value [8]. Multiphasic contrast-enhanced multidetector CT has served as the standard imaging method for the preoperative assessment of both iCCA and pCCA $[9,10]$. It provides a comprehensive evaluation of the primary tumor, the relationship between the primary tumor and adjacent structures such as the hepatic artery or portal vein, and whole abdomen surveillance for potential metastasis [11]. The precontract phase is useful for the detection of intraductal stones and in differentiating stones from tumors. Postcontrast imaging is usually performed at the arterial phase and portal venous phase. The delayed phase, which is obtained 3-5 minutes after contrast medium injection, is useful for the differential diagnosis of iCCA, as iCCA typically shows delayed phase enhancement due to its abundant fibrous stroma [12]. In addition, advanced postprocessing techniques such as three-dimensional reconstruction and maximum intensity projection aid in the accurate assessment of the vascular anatomy and the presence and extent of tumor invasion into the hepatic artery, portal vein, and hepatic parenchyma. In perihilar cholangiocarcinoma, CT has shown a tendency to underestimate the proximal tumor extent, which can be explained by the longitudinal tumor spread along the bile duct wall with microscopic submucosal extension of pCCA [13]. Recently, minimum intensity projection images from the CT data set have been shown to provide CT cholangiography images that may serve as an alternative to endoscopic retrograde cholangiography or MRCP for the assessment of biliary obstruction and longitudinal tumor extent [14]. In recent years, contrast-enhanced MRI with MRCP has been increasingly performed for cholangiocarcinoma, particularly perihilar cholangiocarcinoma.

MRI often includes two-dimensional and three-dimensional (3D) MRCP. Respiratory or navigator triggered 3D isotropic MRCP provides high-spatial-resolution iso-voxel data, as well as maximum intensity projections at multiple projections, which give an excellent overview of the biliary tree and better delineation of fine structures and small pathologies [15]. MRCP is noninvasive and is able to evaluate the whole bile duct, including the most proximal duct to the obstruction and is applicable to patients with prior surgery or anatomic variations. PET scan has limited sensitivity in biliary tumor; however, it may have some utility in the setting of equivocal CT or MRI findings [16]. Routine use of PET scan is not recommended, as it rarely changes surgical management.

\section{Treatment}

Surgical treatment is the preferred option for all subtypes, but, when contemplated, involvement of the vascular structures and lymph nodes needs to be considered. The highly desmoplastic nature of cholangiocarcinoma, its extensive support by a rich tumor microenvironment, and profound genetic heterogeneity, all contribute to its therapeutic resistance. Although surgery and curative liver transplantation are options for selected patients with perihilar cholangiocarcinoma, 5-year survival rates are very low. The chemotherapy regimen of gemcitabine and cisplatin is often used for inoperable disease [17]. The best chance of longterm survival and potential cure is surgical resection with negative margins, but many patients are unresectable due to locally advanced or metastatic disease at diagnosis. As a result of recent efforts, new methods of management have been identified for these patients, including preoperative portal vein embolism and biliary drainage, neoadjuvant chemotherapy with subsequent transplantation, and chemoradiation therapy [18]. Surgical resection for pCCA remains the accepted first line of definitive treatment as long as $\mathrm{R} 0$ resection 
is achieved and adequate future liver remnant is preserved. When evaluating for resectability the capacity to leave adequate hepatic parenchyma is essential. Major and liver-related complications, hepatic dysfunction or insufficiency, longer hospital stays, and 90day mortality are increased when FLR is less than 20\% [19]. Portal vein embolization (PVE) or portal vein ligation (PVL) can be used to increase the FLR by a hemodynamic change and redistribution of growth factors leading to hypertrophy, thus improving the safety of major hepatic resections.

However, it takes 4-6 weeks to wait for the volume of the contralateral liver tissue to increase. Japanese surgeons, who have the most extensive experience of PVE in the setting of pCCA, generally suggest PVE if FLR is less than 40\% [20]. In addition, we must leave enough length for biliary anastomosis. Preoperative biliary obstruction is associated with liver dysfunction and cholangitis, impaired post-operative regeneration and liver failure, and increased risk for perioperative mortality. In our center, biliary decompression is preformed via endoscopic retrograde cholangiopancreatography or percutaneous transhepatic biliary drainage to relieve obstruction and decrease bilirubin. Resection of the involved intra- and extrahepatic bile ducts, as well as the associated hepatic lobe and caudate lobe, is the standard procedure for suitable patients. Survival is highly correlated with resection margin status. Median survival and 5-year survival among patients with a negative margin (R0) resection range from 27 months to 58 months and from $27 \%$ to $45 \%$, respectively. Among patients with a positive microscopic or gross margin, median survival and 5-year survival are significantly worse, ranging from 12 months to 21 months and from $0 \%$ to $23 \%$, respectively [21-26]. At the same time, lymphadenectomy is also performed routinely. Lymph node (LN) metastasis has been shown to be a strong predictor for poor survival outcome. In patients with resectable pCCA, LN metastasis is present in approximately $31-58 \%$ and these patients are reported to have a 5 -year survival rate of $<25 \%[27,28]$.

Perihilar cholangiocarcinoma with invasion of portal vein, hepatic artery or Inferior vena cava was considered a contraindication to surgery traditionally, however, with the improvements in microsurgical techniques, vascular anastomosis and liver transplantation, many outstanding surgeons tend to take more aggressive surgical treatment to achieve R0 margin. Just like the case I presented, we perform a portal vein end-toend anastomosis and remove the diseased part of inferior vena cava, then use an artificial blood vessel to reconstruct Inferior vena cava. Although more and more studies have described acceptable outcomes in patients undergoing vessel resection and reconstruction, these operations should be undertaken by only the most experienced centers with the appropriate hepatobiliary and vascular surgery expertise. Vascular resections should not be performed routinely in pCCA and the decision should be determined intraoperatively based on the judgment of surgeons. The role of neoadjuant chemotherapy in combination with surgical resection for pCCA and iCCA remains undefined. While neoadjuant therapy was reported to have increased the $\mathrm{R} 0$ resection rates in two retrospective analyses, it did not translate into overall survival benefit $[29,30]$. After resection of margin-positive or node-positive pCCA, chemoradiation should be offered to patients. Traditionally considered chemotherapy-resistant, a clinical trial reported a 6-month survival benefit in CCA patients treated with gemcitabine/cisplatin-combination therapy versus gemcitabine monotherapy [31]. Thus patients with advanced and metastatic hilar CC, the standard first-line chemotherapy is gemcitabine and cisplatin. With the advent of targeted immunotherapy, these new treatment methods have shown broad prospects in the treatment of cholangiocarcinoma (Figures 1-5).

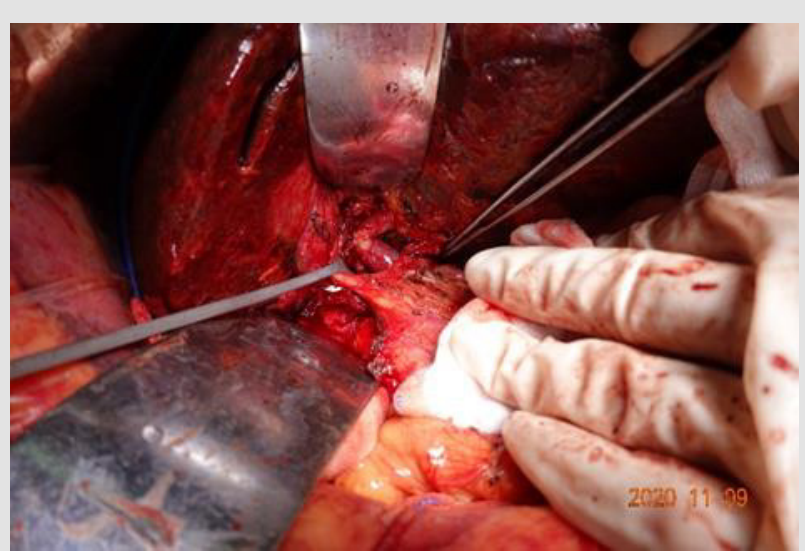

Figure 1.

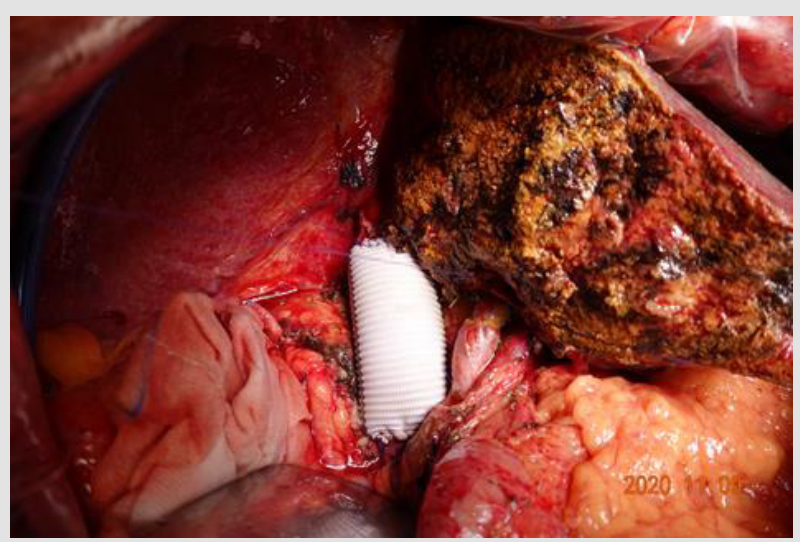

Figure 2. 


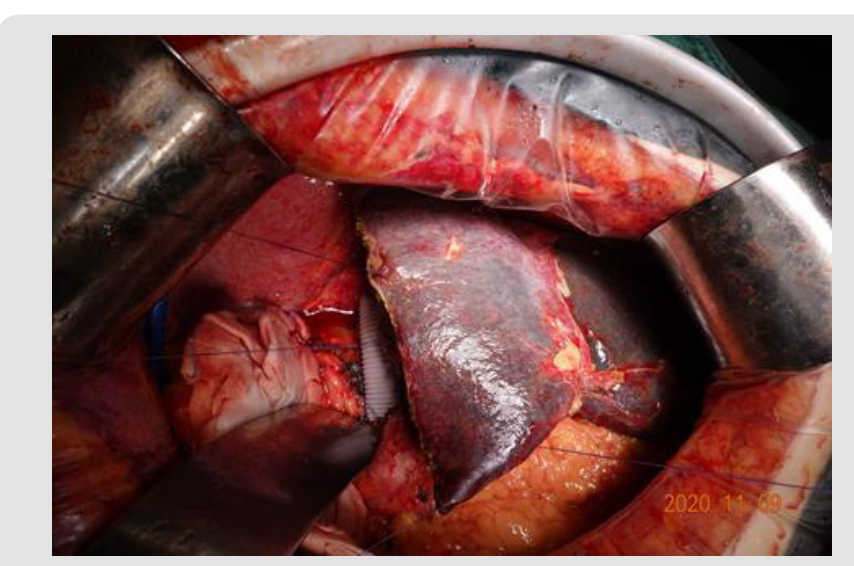

Figure 3.

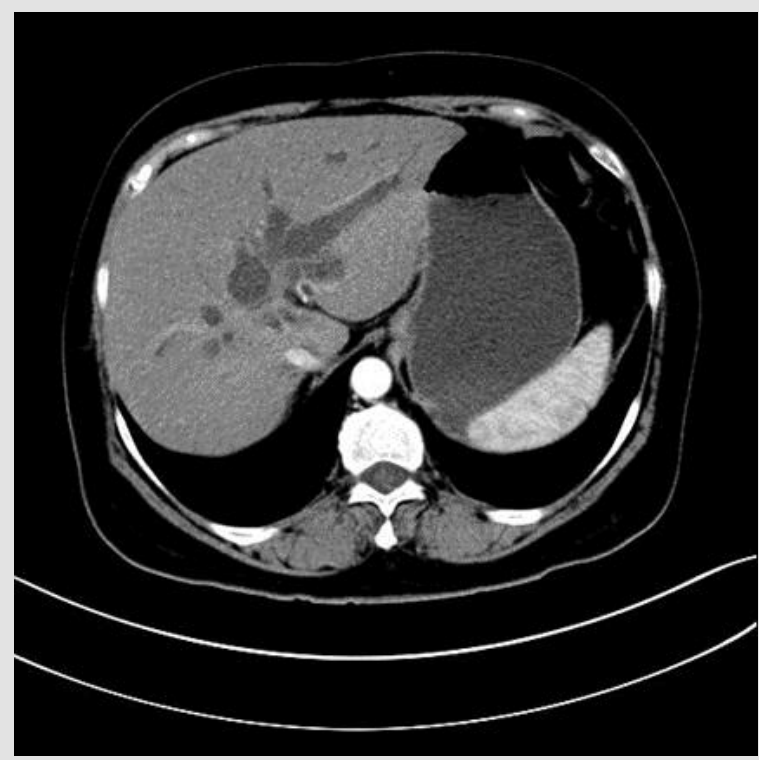

Figure 4 .

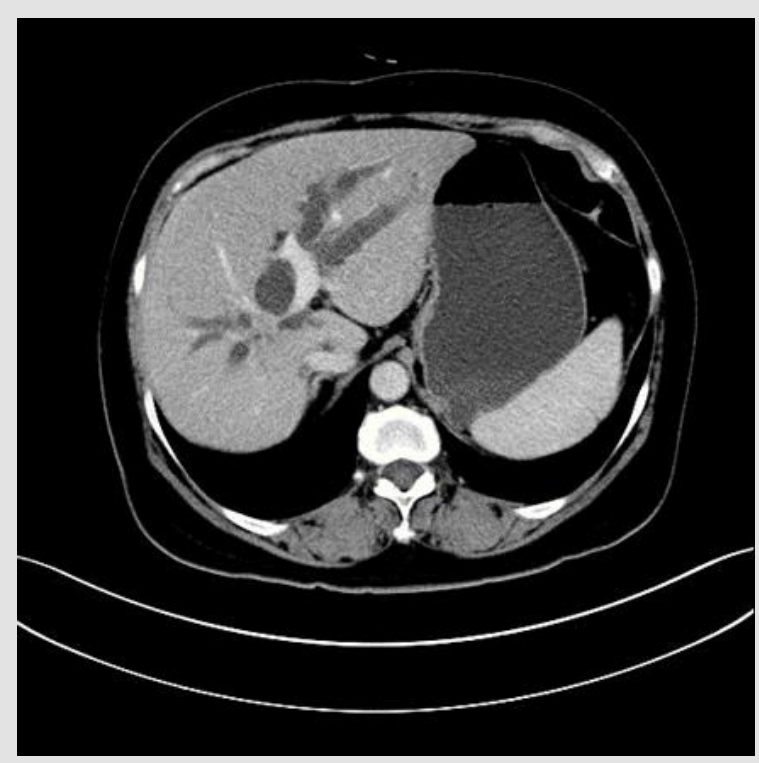

Figure 5.

\section{Conclusion}

Perihilar cholangiocarcinoma is an extremely malignant disease with low 5-year survival rate. Patients present with obstructive jaundice and intrahepatic bile duct dilation. CT and MRI play an important role in evaluation and diagnosis. The only curative treatment is radical resection to achieve negative margin, however, many patients were diagnosed at advanced stage and lost the opportunity to surgery. These patients are candidates for chemotherapy, radiotherapy and other multidisciplinary treatment.

\section{References}

1. N Patel, B Benipal (2019) Incidence of cholangiocarcinoma in the USA from 2001 to 2015: a US cancer statistics analysis of 50 States. Cureus 11(1): e3962.

2. De Oliveira ML, Cunningham SC, Cameron JL, Farin Kamangar, Jordan M Winter, et al. (2007) Cholangiocarcinoma: thirty-one-year experience with 564 patients at a single institution. Ann Surg 245(5): 755-762.

3. Vauthey JN, Blumgart LH (1994) Recent advances in the management of cholangiocarcinomas. Semin Liver Dis 14(2): 109-114.

4. Rizvi S, Khan SA, Hallemeier CL, Kelley RK, Gores GJ (2018) Cholangiocarcinoma - evolving concepts and therapeutic strategies. Nat Rev Clin Oncol 15(2): 95-111.

5. Bismuth H, Corlette MB (1975) Intrahepatic cholangioenteric anastomosis in carcinoma of the hilus of the liver. Surg Gynecol Obstet 140(2): 170-178

6. Deoliveira ML, Schulick RD, Nimura Y, Charles Rosen, Gregory Gores, et al. (2011) New staging system and a registry for perihilar cholangiocarcinoma. Hepatology 53(4): 1363-1371.

7. Tyson GL, El Serag HB (2011) Risk factors for cholangiocarcinoma. Hepatology 54(1): 173-184.

8. Hennedige TP, Neo WT, Venkatesh SK (2014) Imaging of malignancies of the biliary tract- an update. Cancer Imaging 14(1): 14

9. Razumilava N, Gores GJ (2013) Classification, diagnosis, and management of cholangiocarcinoma. Clin Gastroenterol Hepatol 11(1): 13-21.

10. Joo I, Lee JM (2013) Imaging bile duct tumors: pathologic concepts, classification, and early tumor detection. Abdom Imaging 38(6): 13341350 .

11. Blechacz B, Komuta M, Roskams T, Gores GJ (2011) Clinical diagnosis and staging of cholangiocarcinoma. Nat Rev Gastroenterol Hepatol 8(9): 512-522.

12. JM Lee KB, Kyoung Bun Lee, Seung Ho Kim, Soon Ho Yoon, Joon Koo Han, et al. (2011) Intrahepatic mass-forming cholangiocarcinomas: enhancement patterns at multiphasic CT, with special emphasis on arterial enhancement pattern-correlation with clinicopathologic findings. Radiology 260(1): 148-157.

13. Lee HY, Kim SH, Lee JM, Sun Whe Kim, Jin Young Jang, et al. (2006) Preoperative assessment of resectability of hepatic hilar cholangiocarcinoma: combined CT and cholangiography with revised criteria. Radiology 239(1): 113-121.

14. Zandrino F, Benzi L, Ferretti ML, Ferrando R, Reggiani G, et al. (2002) CT cholangiography without biliary contrast agent: technique and initial clinical results in the assessment of patients with biliary obstruction. Eur Radiol 12(5): 1155-1161.

15. Anupindi SA, Victoria T (2008) Magnetic resonance cholangiopancreatography: techniques and applications. Magn Reson Imaging Clin N Am 16(3): 453-466. 
16. Li J, Kuehl H, Grabellus F, Stephan P Müller, Sonia Radunz, et al. (2008) Preoperative assessment of hilar cholangiocarcinoma by dual-modality PET/CT. J Surg Oncol 98(6): 438-443.

17. Razumilava N, Gores GJ (2014) Cholangiocarcinoma. Lancet 383(9935): 2168-2179.

18. Poruk KE, Pawlik TM, Weiss MJ (2015) Perioperative Management of Hilar Cholangiocarcinoma. J Gastrointest Surg 19(10): 1889-1899.

19. Ribero D, Abdalla EK, Madoff DC, M Donadon, E M Loyer, et al. (2007) Portal vein embolization before major hepatectomy and its effects on regeneration, resectability and outcome. Br J Surg 94(11): 1386-1394.

20. Ndoh J, Truty MJ, Aloia TA, Steven A Curley, Giuseppe Zimmitti, et al (2013) Kinetic growth rate after portal vein embolization predicts posthepatectomy outcomes: toward zero liver-related mortality in patients with colorectal liver metastases and small future liver remnant. J Am Coll Surg 216(2): 201-209.

21. Jarnagin WR, Fong Y, DeMatteo RP, Gonen M, Burke EC, et al. (2001) Staging, resectability, and outcome in 225 patients with hilar cholangiocarcinoma. Ann Surg 234(4): 507-517

22. Hasegawa S, Ikai I, Fujii H, Hatano E, Shimahara Y (2007) Surgica resection of hilar cholangiocarcinoma: analysis of survival and postoperative complications. World J Surg 31(6): 1256-1263.

23. Hemming AW, Reed AI, Fujita S, Foley DP, Howard RJ (2005) Surgica management of hilar cholangiocarcinoma. Ann Surg 241(5): 693-699.

24. Hong JC, Jones CM, Duffy JP, Petrowsky H, Farmer DG, et al. (2011) Comparative analysis of resection and liver transplantation for

\section{ISSN: 2574-1241}

DOI: 10.26717/BJSTR.2021.36.005785

Xiangcheng Li. Biomed J Sci \& Tech Res

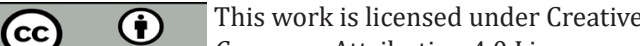
Commons Attribution 4.0 License

Submission Link: https://biomedres.us/submit-manuscript.php intrahepatic and hilar cholangiocarcinoma: a 24-year experience in a single center. Arch Surg 146(6): 683-689.

25. Nishio H, Nagino M, Nimura Y (2005) Surgical management of hilar cholangiocarcinoma: the Nagoya experience. HPB 7(4): 259-262.

26. Nuzzo G, Giuliante F, Ardito F, Giovannini I, Aldrighetti L, et al. (2012) Improvement in perioperative and long-term outcome after surgical treatment of hilar cholangiocarcinoma: results of an Italian multicenter analysis of 440 patients. Arch Surg 147(1): 26-34.

27. S Conci, A Ruzzenente, M Sandri, F Bertuzzo, T Campagnaro, et al. (2017) What is the most accurate lymph node staging method for perihilar cholangiocarcinoma? Comparison of UICC/AJCC $\mathrm{pN}$ stage, number of metastatic lymph nodes, lymph node ratio, and log odds of metastatic lymph nodes. Eur J Surg Oncol 43(4): 743-750.

28. K Mao, J Liu, J Sun, Jianlong Zhang, Jie Chen, et al. (2016) Patterns and prognostic value of lymph node dissection for resected perihilar cholangiocarcinoma. J Gastroenterol Hepatol 31(2): 417-426.

29. JH Jung, HJ Lee, HS Lee, Jung Hyun Jo, In Rae Cho, et al. (2017) Benefit of neoadjuvant concurrent chemoradiotherapy for locally advanced perihilar cholangiocarcinoma. World J Gastroenterol 23(18): 33013308.

30. KM McMasters, TM Tuttle, SD Leach, T Rich, KR Cleary, et al. (1997) Neoadjuvant chemoradiation for extrahepatic cholangiocarcinoma. Am J Surg 174(6): 605-608.

31. Valle J, Wasan H, Palmer DH, David Cunningham, Alan Anthoney, et al (2010) Cisplatin plus gemcitabine versus gemcitabine for biliary tract cancer. N Engl J Med 362: 1273-1281.

$\begin{array}{ll}\text { BIOMEDICAL } & \text { Assets of Publishing with us } \\ \text { RESEARCHES } & \text { - Global archiving of articles } \\ \text { - Immediate, unrestricted online access }\end{array}$

